\title{
Textual notes
}

\section{Abbreviations}

\section{Pre-modern editions of Wang Wei's works}

GKJ: Tang Wang Youcheng shi ji zhu shuo 唐王右丞詩集註說. Compiled by Gu Kejiu 顧可久. Preface dated 1560; follows the structure of LCW. Reprinted 1590.

LCW: Xuxi xiansheng jiao ben Tang Wang Youcheng ji 須溪先生校本唐 王右丞記 Complied by Liu Chenweng 劉辰翁 with critical comments. Yuan edition. Includes only poetry. Evidently based on the Masha edition (SGTB), but collated with something else. Reprinted in 1504 .

LMC: Wang Mojie shi ji 王摩詰詩集. Compiled by Ling Mengchu 凌濛初. No date; late Ming.

QTS: Quan Tang shi 全唐詩. 1707, with later revisions. Poem order and variants suggest sources considerably at odds from other surviving editions.

QTW: Quan Tang wen 全唐文. 1814.

QZZ: Lei jian Tang Wang Youcheng ji (published by Gu shi Qizizhai) 類箋唐王右丞集（顧氏奇字齋刊）. Compiled by Gu Qijing 顧起經. Preface dated 1555. Earliest suriving edition to collate earlier editions.

SGTB: Wang Youcheng wen ji (Qing Qian shi Shugutang yingchao) 王右丞文集（清錢氏述古堂影沙）. This is a facsimile of the Masha 麻沙 Song edition (which only survives in a Japanese collection). Its organization is the same as SSB, but there are enough variant readings to suggest that the Masha edition also incorporated readings from a variant textual line. Includes prose.

SSB: Wang Mojie wen ji (Song Shu ben) 王摩詰文集（宋蜀本） Published in Shu, probably Northern Song. Includes prose. Earliest surviving edition.

WMJJ: Wang Mojie ji 王摩詰集. 10 juan. No date or name Includes poetry and prose. Earliest surviving collection to organize texts by metrical genre; order of poems suggests it derives mostly from the Masha textual lineage. Probably printed in first half of sixteenth 
century. This edition was the basis for various Ming reprints, including compendia of Tang poets.

ZDC: Wang Youcheng ji jian zhu. 王右丞集箋注. Compiled by Zhao Diancheng 趙殿成. Preface dated 1737. Zhao notes that he collated from LCW, GKJ, LMC, and QZZ. This is the base edition for this translation.

\section{Other sources for Wang Wei texts}

GXJ: Guo xiu ji 國秀集. Compiled by Rui Tingzhang 䓎挺章. C. 740s. HYYLJ: Heyue yingling $j i$ 河获英露集. Compiled by Yin Fan 殷璠. C. 753 .

TSJS: Tang shi ji shi 唐詩紀事. Compiled by Ji Yougong 計有功. Mid twelfth century.

TSPH Tang shi pin hui 唐詩品鱼. Compiled by Gao Bing 高鿇. Late 1300s.

TWC: Tang wen cui 唐文粹. Compiled by Yao Xuan 姚鉉. Completed in 1011 , printed in 1039 .

WSTR: Wan shou Tang ren jueju 萬首唐人絕句. Compiled by Hong Mai 洪递. Presented to throne 1192.

WYYH: Wenyuan yinghua 文苑英華. Finished 987, with later supplements and corrections.

YFSJ: Yuefu shi ji. Compiled by Guo Maoqian 郭茂倩. Twelfth century. YKLS: Yingkui lüsui 瀛奎律髓. Compiled by Fang Hui 方回. 1282.

11.2.8: 去: GKJ, LMC have 出.

11.2.11: Supplying 表 from WMJJ, QZZ, and QTS for ZDC 漢.

11.3.3: 戶5: WYYH, QTS have 井.

11.3.8: 妙: WYYH has 妰.

11.4.5: 比: SSB, QTS have 此.

11.4.10: Supplying 留 from SSB, SGTB, and LCW for ZDC 流.

11.5.13: 喜: WYYH, QTS have 佳.

11.6.4: 龍: LMC has 金.

11.8.11: 戰: SSB has 物. 
11.9.9: $\quad$ Supplying 䈍 from QZZ, LMC, and QTS for ZDC 樂.

11.9.12: ～紀: SSB, WMJJ have 紹; QZZ, LMC have 治.

11.10.11: 定: WYYH has 止.

11.11: $\quad$ title: Supplying 官 from SGTB and QTS for ZDC 臣.

11.11.3: I: SSB, SGTB, WMJJ have 生. 逢: QTS has 無, SSB has 逸.

11.14.3: 裹: QTS has 外.

11.14.6: Supplying 太- from SSB, SGTB, and LCW for ZDC 太乙.

11.14.8: 有: QTS has 見.

11.16.2: 自: WYYH has 復.

11.19.9: Supplying 路 from SSB and QTS for ZDC 露.

11.20: title: Following SSB, WMJJ, and QTS in removing 等 at the end.

11.21.2: 苗: WMJJ, QZZ, LMC have 田.

11.21.9: Supplying 夕 from QTS for ZDC 多. Supplying 拆 from SGTB, LCW, and QTS for ZDC 折.

11.24a: Supplying title from QTS.

11.24b: Supplying title from QTS.

11.24c: Supplying title from QTS.

12.1.1: 槿: LCW has 種. 。故: SGTB, LCW have 外.

12.1.2: 今: SSB, WMJJ, QTS have 新.

12.1.7: 畫: WMJJ has 書.

12.2.9: 醥: SGTB has 酷.

12.2.16: 山: SSB, WMJJ, WYYH have 川.

12.2.17: 為: SSB, WYYH have 若.

\section{3 preface:}

1. 1 :

峴: SSB has 見. 服: SSB, WMJJ, QTS has 格.

1. 26: Supplying 稽首 fromQTS for ZDC 馳首. SSB, SGTB, WMJJ have 地首.

1. 36: Supplying 布 from SSB for ZDC 恢.

12.4: title: 徐: SSB, WMJJ, QZZ have 禰.

12.4.1: 草色: QZZ has 色早. 
12.4.12: $\quad$ 絕: SSB, WMJJ, QZZ have 䋓.

12.7.2: 地: LMC has 殿.

12.7.8: 鳴: SSB, WYYH, QTS have 明.

12.7.12: 踏: WYYH has 蹈.

12.7.18: 緣: SSB has 紫. Supplying 简蕉 from WYYH and QTS for ZDC 芼慈.

12.8.2: 開: WYYH has 衣.

12.8.8: 紅: TSJS, LMC have 青.

12.8.17: 諸: WYYH, TSJS, SSB have 朱.

12.8.20: 露: SSB has 露.

12.8.23: 知: SSB has 言.

12.10.4: 雞: TSPH has 禽.

12.10.6: 杪: LMC has 上.

12.10.11: Supplying 多 from SGTB, WYYH, and QTS for ZDC 諳.

12.11.1-2: For these two lines, WYYH and QTS have 玉壱何用好, 偏許素冰居.

12.11.11-12: For these two lines, WYYH has 若向食夫比, 貞心定不如.

12.12.4: 君: WYYH has 公.

12.12.5: Supplying 催 from WYYH for ZDC 隨.

12.12.6: 拜: WYYH has 問.

12.13.6: Supplying 聞 from QTS for ZDC 開.

12.15: title: SSB has 過沈居哭沈居士; SGTB has 過沈居士山居 哭沈居士.

12.15.5: 雀: QTS has 鵲.

12.16.1: 當: LCW, WMJJ, QTS have 嘗; SSB, SGTB have 常.

12.16.4: 至: WMJJ, QTS have 主.

12.16.20: Supplying 底 from WYYH and QTS for ZDC 何. Supplying 碎 from WYYH and QTS for ZDC 與.

12.16.23: 城: QTS has 域.

12.16.25: 見: WYYH has 在.

12.16.34: Supplying 開 from SSB, SGTB, LCW, and WYYH for ZDC 聞.

12.16.36: 天: LMC has 問. 
12.16.41: 終: QTS has 難.

12.16.42: 泥: SGTB, LCW, WYYH have 哭.

12.16.64: 斷: QTS has 繼.

13.1: title: Taken from QTS, instead of ZDC 答裴迪. WSTR has 答裴迪憶鐘南山。

13.2: title: WSTR is missing 諸; LMC is missing 妹.

13.3: title: WSTR has 聞裴迪吟詩戲贈.

13.3.2: $\quad$ 悲: LMC has 愁.

13.4.4: 令: SSB has 暮.

13.5.3: 驚: SSB has 空.

13.7.2: 清: GKJ has 晴. 浦: SSB, WSTR, QTS have 蒲.

13.9.4: 掊復: QZZ, LMC have 復挬.

13.15.3: 翠: SSB has 峯.

13.16.4: Supplying 芙蓉 from SSB, WMJJ, and QTS for ZDC 荣莫.

13.18.1: 上: WSTR has 仙.

13.20.3: 首: SGTB, LCW have 看.

13.20.4: 山青: SSB, WMJJ, QTS have 青山.

13.23.3: 翔: SSB, WMJJ, QTS have 腤.

13.24.2: 向: TSJS has 尚.

13.27.4: 紛紛: SGTB, LCW, GKJ have 絲絲.

13.28.3: 偶: GKJ, LMC have 准.

13.29.4: 君: SGTB, LCW, GKJ have 身.

13.30: title: WSTR lacks 臨高臺.

13.31: title: Supplying full title from SSB, WSTR, and TSPH from ZDC 送別.

13.31.3: 明年: WMJJ, QZZ have 年年.

13.32a: title: Taken from QTS.

13.33a: title: Taken from QTS.

13.33b: $\quad$ Poem added from QTS.

13.34: title: HYYLJ has 息夫人怨; QXJ has 息姷怨. 
13.34.2: 能忘: BSS has 䆓忘; SSB, WSTR, TSJS, QTS have 難忘; YFSH has 寧無. 蕾: GXJ has 昔, TSJS has 買.

13.35-37: title: HYYLJ, TWC have 婕妤怨. GXJ anthologizes the third poem under the title 扶南曲.

13.35.3: 愇: QTS has 惟.

13.35.4: Supplying 不 from YFSJ, SSB, SGTB, and WMJJ for ZDC 明.

13.36.2: 恩: WYYH has 寵.

13.37.3: 向: GXJ has 在.

13.37.4: 笑語: GXJ, YFSJ, SSB, SGTB have 語笑.

13.38.2: 持: SSB, WMJJ, QTS have 時.

13.40: title: 梨花: WYYH has 海棠花; SSB, SGTB have the title 梨花詠.

13.40.4: 入: WYYH has 向.

13.40a: title: Taken from QTS.

13.40b: title: Taken from QTS.

13.41: title: WSTR has 菩提寺禁示裴迪; QTS has 菩提寺禁口號 又示裴迪.

13.41.1: 應: QTS has 羅.

13.41.4: 向: WSTR has 去.

13.42.3: 船: SSB, SGTB have 舡.

13.44.3: 愁心: SSB, SGTB, WMJJ have 心心.

13.44.4: Supplying 階前 from SSB, LCW, WMJJ, and WSTR for ZDC 玉階.

13.45: title: 詠 supplied from SSB, SGTB, WMJJ, and TSJS. TSJS adds 與 at beginning of title.

13.46: title: SSB has 荣莫詠.

13.46.1: 朱實: QZZ has 茉莫.

13.46.3: Supplying 與 from SSB, SGTB, and QTS for ZDC 有.

13.47: title: WSTR has 哭孟襄陽; TSJS has 憶孟. Note added from SSB, SGTB, and QTS.

13.47.1-2: TSJS has 故人今不見, 日夕漢江流. 
14.1.1: 出入: SSB, WMJJ, QZZ have 厭見.

14.1.3: 蹀婜: SSB, WMJJ, QZZ have 官府.

14.3.2: 村: SSB, QZZ have 林.

14.4.1: ZDC supplies 芳 for 春 based on TSPH. 春: SSB, QTS have 秋. 綠: LMC has 碧.

14.4.4: 不: WSTR has 未.

14.6.1: 宿: WSTR has 夜.

14.6.2: 春: QTS has 朝.

14.6.4: 鶯: LMC has 鳥.

14.7.4: 東谷: SSB, SGTB have 舍; LMC has 西舍.

14.8.2: 多: WSTR has 皆.

14.8.3: 意氧: WSTR has 氧味.

14.10.2: 重: YFSJ has 群.

14.14.2: 佳: SSB, SGTB have 嘉.

14.15.2: 樹: LMC has 枝. 梢: WMJJ, QZZ, QTS have 披.

14.16.1: 臨: QZZ, LMC have 鄰.

14.17.3: 松: WYYH has 林.

14.17.4: For this line SSB has 白眼看君是甚人.

14.7a: $\quad$ title: Taken from QTS.

14.7b: $\quad$ title: Taken from QTS.

14.7c: $\quad$ title: Taken from QTS.

14.18: $\quad$ title: QZZ and GKJ lack 拜掃.

14.19: title: YFSJ, QTS have 渭城曲.

14.19.2: 柳色: QTS has 楊柳. 新: SSB, WSTR, YFSJ have 春.

14.20: title: Supplying title from WSTR and QTS from ZDC 送別. QTS title has 二 for 三.

14.23: title: 沈子福: WMJJ, QZZ, QTS have 沈子. 歸: WSTR, TSPH have 之.

14.26.2: 官: SSB, TSJS have 寮; QTS has 僚. 再: SSB, SGTB, TSJS have 更.

14.26.3: 空: TSJS has 深. 
14.27.2: Supplying 烽 from SSB for ZDC 峯.

14.28: title: Supplied from GXJ and QTS for ZDC 哭殷遙.

14.29.4: 銷: SSB has 消.

15.1: WYYH: attrib. Wang Wei; TWC: attrib. Wang Changling; QTS: attrib. both.

15.1.9: 清澄: TWC has 澄清; QTS has 清燈.

15.1.10: 破影: TWC has 影破.

15.2.5: 雲: QTS has 嚴。

15.3.1: 欲: YKLS, QTS have 復.

15.3.3: 長: QTS has 良.

15.4-8: QZZ: included in waibian; SSB, SGTB: missing. WYYH, QTS: attrib. Sun Ti.

15.9: QZZ: waibian; missing from other editions. WYYH, QTS: attrib. Song Zhiwen.

15.12-13: YFSJ, WMJJ, QZZ, LMC: attrib. Wang Wei; WSTR, TSJS, QTS: attrib. Wang Ya.

15.14-15: YFSJ, WMJJ, QZZ, LMC: attrib. Wang Wei; WSTR, QTS: attrib. Wang Ya; TSJS: attrib. Zhang Zhongsu 張仲素.

15.15.1: 無: YFSJ, TSJS, LMC have 何.

15.16: WSTR, TSJS: attrib. Wang Wei. QZZ, LMC: included in waibian.

15.16: title: LMC: 江上贈李急年.

15.17-18: YFSJ, WMJJ, QZZ, LMC attrib. Wang Wei. WSTR: attrib. Wang Ya. TSJS: attrib. Zhang Zhongsu. QTS: 15.17 attrib. Wang Ya, 15.18 attrib. Zhang Zhongsu.

15.17-18: title: 樂: SSB, WMJJ, QZZ, LMC have 墑.

15.17.1: 和: QZZ has 何.

15.17.3: 探: TSJS has 採.

15.18.2: 威: TSJS has 風.

15.19: WMJJ, QZZ, LMC attrib. Wang Wei. SSB, WSTR, QTS attrib. Wang Ya. TSJS attrib. Zhang Zhongsu. 
15.21-22: YFSJ, WMJJ, QZZ, LMC attrib. Wang Wei. WSTR, QTS attrib. Wang Ya. TSJS: 15.21 attrib. Zhang Zhongsu, 15.22 attrib. Wang Ya.

15.21-22: title: TSJS has 平戎詞.

15.23: WMJJ, QZZ, LMC attrib. Wang Wei. SSB, WSTR, TSJS, QTS attrib. Wang Ya.

15.23.1: 羽: WSTR has 箭.

15.24-28: WMJJ, QZZ, LMC attrib. Wang Wei. SSB, WSTR, QTS attrib. Wang Ya. TSJS attrib. Wang Ya, omits \#3, gives 閏思 as title for \#5. Title: 贈: TSJS has 寄.

15.29: QZZ: included in waibian. Originally attributed to Wang Wei in the WSTR, actually the first four lines of a Meng Haoran regulated verse (過故人莊).

15.30: QZZ: included in waibian. TSJI, QTS attrib. Zhao Gu.

15.31-32: YFSJ, WMJJ, QZZ, LMC: attrib. Wang Wei; WSTR, TSJS, QTS: attrib. Wang Ya.

15.32.1: 貕: YFSJ, TSJS have 蹊.

15.32.2: 春: YFSJ has 風.

15.33-34: YFSJ, WMJJ, QZZ, LMC: attrib. Wang Wei; WSTR, TSJS, QTS: attrib. Wang Ya.

15.33.2: 生: WSTR, YFSJ, TSJS have 長.

15.34.2: 消: YFSJ, LMC have 清.

15.35-36: YFSJ, WMJJ, QZZ, LMC attrib. Wang Wei. WSTR attrib. Wang Wei and Wang Ya (anthologized twice). TSJS attrib. Zhang Zhongsu. QTS: 15.35 attrib. Zhang Zhongsu, 15.36 attrib. Wang Ya.

15.35.2: 陰: WSTR, YFSJ, TSJS have 雲.

15.35.3: 㣂: YFSJ, LMC have 壁.

15.36: title: TSJS has 春閏怨.

15.37: YFSJ, WMJJ, QZZ, LMC: attrib. Wang Wei; WSTR, TSJS, QTS: attrib. Wang Ya.

15.38-39: YFSJ, WMJJ, QZZ, LMC: attrib. Wang Wei; WSTR, TSJS, QTS: attrib. Wang Ya. 
15.38.3: 愁: YFSJ, TSJS, LMC have 秋.

15.38.4: 驚: TSJS has 督.

15.40-41: YFSJ, WMJJ, QZZ, LMC attrib. Wang Wei. WSTR attrib. Wang Ya. TSJS, QTS: 15.40 attrib. Wang Ya, 15.41 attrib. Zhang Zhongsu.

15.40.1: 漢: TSJS has 發; WSTR, WMJJ have 後.

15.40.4: 從: YFSJ has 君.

15.42: WMJJ, QZZ, LMC attrib. Wang Wei. SSB, WSTR, QTS attrib. Wang Ya. TSJS attrib. Zhang Zhongsu.

15.42.1: 遥: TSJS, WSTR have 遊.

15.42.2: 挽: TSJS, SSB have 惹.

15.42.3: 遍: WSTR, LMC, SSB have 㿿.

15.43-44: WMJJ, QZZ, LMC attrib. Wang Wei. SSB, WSTR, QTS attrib. Wang Ya. TSJS attrib. Zhang Zhongsu.

15.43.2: 關: TSJS has 江.

15.45: WMJJ, QZZ, LMC attrib. Wang Wei. SSB, WSTR, TSJS, QTS attrib. Wang Ya.

15.45.1: 殿: TSJS has 觀.

15.46: title: WSTR, QZZ have 李烏年所歌; LMC has 雜詩; YFSJ has 伊州第一叠; QTS has 伊洲歌.

15.46.1: 清: YFSJ, LMC have 秋. 明: WSTR has 朗. 若相思: YFSJ has 獨離居.

15.46.2: 我: LMC has 軍.

15.46.4: 寄: WSTR, TSJS LMC have 附.

16.1.5: $\quad$ 鳥: WYYH has 禽.

16.1.9: 妖: QTW has 伎.

16.1.11: 喬: SGTB has 高. 以: WYYH has 于.

16.1.12: 以: WYYH has 于.

16.1.16: 而: TWC has 之.

16.1.24: 願: WYYH has 每.

16.1.26: Supplying 恩 from QTW for ZDC 思. 無: WYYH has 何.

16.1.36: 筐: WYYH has 筒; TWC has 筐.

16.1.38: 寶: WYYH has 瑤.

16.1.39: 羽毛: WYYH has 毛羽. 
16.1.47: 其: WYYH, QTW have 於.

16.2.1: Supplying 某 from SSB, SGTB for ZDC 維 (Chen Tiemin suggests this is the proper reading since the memorial is presented on behalf of a group of ministers, not Wang Wei alone).

16.2.5: Supplying 曾於九疑山 from QTW for ZDC 曾九疑山於.

17.3.16: 妓: QTW has 伎.

17.3.18: 頻: SSB has 類.

17.5.6: 大聖 supplied for QTW.

17.5.7: Supplying 弘 from SSB, SGTB for ZDC 宏.

17.5.12: 人: SSB, SGTB, WMJJ have 麻.

17.5.19: 仁王: QTW has 神王.

17.5.20: Supplying 今 from SGTB, QTW for ZDC 令.

17.6.5: Supplying 文武大聖孝感 from QTW.

17.6.8: 國: SSB, SGTB have 圖.

17.6.19: 偶: QTW has 遇.

17.7.11: Supplying 請 from SSB, SGTB for ZDC 謝.

17.8.2: 能: WYYH has 壽.

17.8.3: 䑨: WYYH has 競.

17.8.6: 洗: SSB, SGTB, WMJJ, QZZ have 失.

17.8.8: 明: WYYH has 聖.

17.8.10: 始: WYYH has 昔.

17.8.11: $\quad$ SSB omits 又.

17.8.13: 籍以: WYYH has 籍見.

17.8.17: 歷: WYYH has 効. 甚: SSB has 其.

17.8.19: 繫: WYYH has 狀.

17.8.20: 憐: WYYH has 恤.

17.8.26: 弟: SSB has 着.

17.8.27: 妨: WYYH has 其.

17.8.34: 廷: WYYH has 行.

17.9.3: 樹: SSB has 聞.

17.9.14: 天: SSB has 大.

18.5.1: 將軍車騎: SSB has 將軍騎; SGTB has 將多車騎.

18.5.7: $\quad$ Supplying F from SSB and QTW for ZDC 不. 
18.5.8: 以: SGTB has 比.

18.5.15: Supplying 完 from SSB for ZDC 治. Supplying 亲 from SSB for ZDC 兵.

18.5.17: Supplying 辰 from SSB and SGTB for ZDC 白.

18.6.2: $\quad$ Supplying 獨 from SSB.

18.6.3: Supplying 比 from SSB, SGTB for ZDC 北.

18.6.6: 村: SSB has 社. 問: SSB, SGTB have 聞.

18.7.7: 淡: SSB, SGTB have 啖. 霞霖: SGTB has 霖雨.

18.7.17: $\quad$ SSB omits 樵蘇.

18.7.20: Supplying 跼 from SGTB, WMJJ, and QTW for ZDC 踞.

18.7.23: 愛: SGTB has 憘.

18.7.31: 自: QTW has 是.

18.7.40: Supplying 當 from SSB for ZDC 嘗; SGTB, WMJJ have 常.

18.7.51: 博: SSB has 賑; 苟: SSB has 自.

18.7.55: SGTB adds concluding phrase 所維白.

18.8.4: 閉: SSB, SGTB, WMJJ have 開. 地: SSB has 遥.

18.8.6: 忘: WMJJ has 存.

18.8.10: 䩸䩬: QTW has 煜煜.

19.1.11: 典邦教: TWC has 掌邦典.

19.1.14: 合: SGTB has 食.

19.1.15: 選: SGTB, TWC, QTW have 撰.

19.1.16: 谷 supplied from QTW.

19.1.17: 屚山: SSB has 遠.

19.1.18: 其: TWC, QTW have 于. 源于: TWC, QTW have 環其.

19.1.19: 家: WMJJ has 冢; QTW has 泉.

19.1.21: 卉木: SSB has 丹木; TWC, QTW have 芳去.

19.1.22: 花: TWC, QTW have 桃. 㯈渏: SGTB, TWC have 超忽.

19.1.32: 合 supplied from SSB, SGTB, and WMJJ.

19.1.33: 賢: TWC, QTW have 思.

19.1.35: SSB, SGTB add 云云 at end.

19.5.4: 中: QTW has 門.

19.7.1: ZDC thinks 上 is a miscopying of 山 or. 
19.10.4: $\quad$ Supplying 者 from SGTB (missing in ZDC).

19.10.5: 陰陽: SSB, SGTB have 強陽.

20.1.2: Supplying 度 from SGTB, WMJJ, and QTW for ZDC 庶. 大: SSB has 天.

20.1.13: 願: SSB has 賴.

20.1.16: 薩: SSB has 提.

20.1.24: 中: SSB, SGTB, WMJJ have 尊.

20.1.26: Supplying 功 from QTW to fill in ZDC lacuna.

20.2.2: 㴚: SSB, WMJJ have 垢.

20.2.4: 心: SSB has 子.

20.2.12: Supplying 望 from SGTB for ZDC 至.

20.2.16: 樂: SGTB has 尊.

20.3.1: 得: SSB, SGTB, WMJJ have 碍.

20.3.5: $\quad$ Supplying - from QTW.

20.3.6: 真: SGTB has 無.

20.3.11: 百: SGTB, WMJJ have 上.

20.3:

gatha 5: Supplying first 空 from SSB, SGTB, and QTW for ZDC 定.

20.4.7: 理: QTW has 倫. Supplying 侯 from SSB and SGTB for ZDC 侯. QTW has 仁.

20.4.13: 讚: SGTB has 偈.

24.1.1: $\quad$ Supplying 得 from SSB for ZDC 旱.

24.1.2: 萬: SSB has 百.

24.1.11: Supplying 固from SSB and SGTB for ZDC 同.

24.1.16: 餬: SSB has 飲.

24.1.21: Supplying 焚 from SSB, WMJJ, QZZ, and QTW for ZDC 聞.

24.1.23: 賾: Correction from ZDC 頣. 履: SSB has 屡.

24.1.29: 跑: SGTB, QTW have 跪.

24.1.34: 周密: SSB has 虫米; SGTB, WMJJ have 由米.

24.1.47: Supplying 為其 from SSB for ZDC 共為. SGTB, WMJJ, QZZ have 為共.

24.1.48: Supplying 亞 from SSB, WMJJ, and QTW for ZDC 詎. 24.1.49: 棌: SSB, WMJJ have 是.

24.1.51: Supplying 祜 from SSB and WMJJ for ZDC 峴. 
25.1: title: TWC, QTW have 六祖能禪師碑銘; 並序: supplied from SSB, SGTB, and WMJJ; SSB and SGTB add phrase 為人作.

25.1.6: 准: SGTB has 推.

25.1.30: 徒: SSB, SGTB, QTW have 其.

25.1.59: First 人: QTW has 天.

25.2.2: 流: SSB has 雄. 若: SSB has 者.

25.2.5: 中 added from SSB, QTW. 其: missing from QTW.

25.2.6: $\quad$ Supplying 去 from SSB, SGTB, and WMJJ for ZDC 玄. QTW has 走.

25.2.14: 指: SSB has 恉.

25.2.15: 五十二: QTW has 五十一. 凡三十二 missing from ZDC, supplied from SSB, SGTB, WMJJ. QTW has 二十二 for 三十二. 